\section{Ultrasound findings in peliosis hepatis}

\author{
Yi Dong ${ }^{1}$, Wen-Ping Wang ${ }^{1}$, Adrian Lim² ${ }^{2}$ Won Jae Lee ${ }^{3,4}$, Dirk-Andre Clevert ${ }^{5}$, \\ Michael Höpfner ${ }^{6}$, Andrea Tannapfel ${ }^{7}$, Christoph Frank Dietrich ${ }^{8}$ \\ 'Department of Ultrasound, Zhongshan Hospital, Fudan University, Shanghai, China; \\ ${ }^{2}$ Department of Imaging, Imperial College London and Healthcare NHS Trust, Charing Cross \\ Hospital Campus, London, UK; ${ }^{3}$ Department of Radiology and Center for Imaging Science, \\ Samsung Medical Center, Sungkyunkwan University School of Medicine, Seoul; ${ }^{4}$ Department \\ of Health Science and Technology and Medical Device Management and Research, Samsung \\ Advanced Institute for Health Science and Technology, Sungkyunkwan University, Seoul, \\ Korea; ${ }^{5}$ Interdisciplinary Ultrasound-Center, Department of Radiology, University of Munich- \\ Grosshadern Campus, Munich; ${ }^{6}$ Department Gastroenterologie, Klinik für Innere Medizin, \\ Agaplesion Diakonie Kliniken Kassel, Kassel; ${ }^{7}$ Institut für Pathologie, Ruhr-Universität, \\ Bochum, Germany; ${ }^{8}$ Department Allgemeine Innere Medizin (DAIM), Kliniken Beau Site, \\ Salem und Permanence, Hirslanden, Bern, Switzerland
}

Purpose: The aim of this study was to retrospectively evaluate contrast-enhanced ultrasound (CEUS) findings in patients with peliosis hepatis (PH).

Methods: A retrospective analysis was conducted of CEUS features in 24 patients with histopathologically confirmed PH (11 men and 13 women; mean age, 32.4 \pm .1 years; range, 28 to 41 years). All lesions were histologically proven, either by core needle biopsy $(n=10)$ or by hepatic surgery $(n=14)$.

Results: The mean size was $36.8 \pm 12.4 \mathrm{~mm}$ (range, 10 to $80 \mathrm{~mm}$ ). On B-mode ultrasonography (BMUS), all PH lesions were heterogeneously hypoechoic, with well-defined margins but irregular shapes. No mass effect was observed. During the arterial phase of CEUS, all lesions displayed mild heterogeneous hyperenhancement $(83.3 \%, 20 / 24)$ or isoenhancement $(16.7 \%, 4 / 24)$. Furthermore, $87.5 \%$ of the $\mathrm{PH}$ lesions showed mild washout after 1 minute in the portal venous phase (30-120 seconds) and mild washout in the late phase (>120 seconds).

Conclusion: The lack of a mass effect on BMUS, mild heterogeneous arterial hyperenhancement, and washout in the very late portal venous phase (after 1 minute) on CEUS are characteristic of $\mathrm{PH}$. Although it is a histological diagnosis, PH should be considered in the differential diagnosis when the clinical context does not favor a malignancy or infection.

Keywords: Focal peliosis hepatis; Contrast-enhanced ultrasound; Focal liver lesions

\section{Introduction}

Peliosis hepatis (PH) is a rare benign disease, which is histologically characterized by blood-filled cystic cavities of various sizes and irregular shapes. These cavities communicate with the hepatic

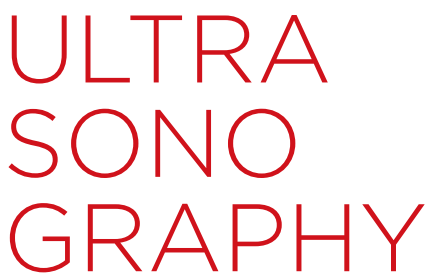

\section{ORIGINAL ARTICLE}

https://doi.org/10.14366/usg.20162 pISSN: 2288-5919 e elSSN: 2288-5943 Ultrasonography 2021;40:546-554

Received: October 10, 2020

Revised: February 20, 2021

Accepted: February 22, 2021

Correspondence to: Christoph Frank Dietrich, MD, PhD, MBA, Department Allgemeine Innere Medizin (DAIM), Kliniken Beau Site, Salem und Permanence, Hirslanden, 3036 Bern, Switzerland

Tel. +41-79-834-7180

Fax. +41-31-337-6000 E-mail: c.f.dietrich@googlemail.com

This is an Open Access article distributed under the terms of the Creative Commons Attribution NonCommercial License (http://creativecommons.org/ licenses/by-nc/4.0/) which permits unrestricted noncommercial use, distribution, and reproduction in any medium, provided the original work is properly cited.

Copyright @ 2021 Korean Society of Ultrasound in Medicine (KSUM)

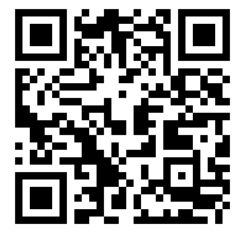

How to cite this article:

Dong Y, Wang WP, Lim A, Lee WJ, Clevert DA, Höpfner $M$, et al. Ultrasound findings in peliosis hepatis. Ultrasonography. 2021 0ct:40(4):546-554. 
sinusoids and are associated with rupture of the reticulin framework [1]. PH has been described as typically involving the entire liver [2], and with the progress of modern medical imaging technologies, $\mathrm{PH}$ is now more frequently encountered in clinical practice [3].

$\mathrm{PH}$ is often asymptomatic; however, it may be associated with rare, but severe complications, which include hepatic failure, portal hypertension, and liver rupture leading to hemoperitoneum and hemodynamic instability [2]. In clinical practice, an accurate preoperative diagnosis of $\mathrm{PH}$ can have an impact on the immediate management of patients and help avoid unnecessary surgery or biopsy, thereby reducing morbidity. Withdrawal of the causative toxin or introduction of specific treatment (such as antibiotics in bacillary peliosis [4], particularly in immunocompromised patients), can resolve the disease and prevent serious complications such as hepatic failure or death owing to intra-abdominal hemorrhage [5].

Most cases of PH are detected incidentally and have no symptoms. The imaging findings of $\mathrm{PH}$ are often nonspecific and vary depending on the different pathological patterns of the disease and presence of hepatic steatosis. The characteristics of $\mathrm{PH}$ on abdominal ultrasonography, computed tomography (CT), magnetic resonance imaging (MRI), angiography, and fludeoxyglucose positron emission tomography/CT have only been sporadically described in case reports $[6,7]$. The nonspecific imaging characteristics of $\mathrm{PH}$ have a broad spectrum and are often misleading [8]. Typically, it is difficult to clearly distinguish PH from other hypervascular lesions on imaging studies alone [1], and a histopathological evaluation is thus necessary. There is, however, an increased risk of bleeding with needle biopsy of vascular lesions [9] and therefore, the understanding of the imaging features of PH may be helpful for establishing the correct diagnosis, occasionally without the need for sampling.

According to the current European Federation of Societies for Ultrasound in Medicine and Biology (EFSUMB) and World Federation of Societies for Ultrasound in Medicine and Biology (WFUMB) guidelines, contrast-enhanced ultrasonography (CEUS) as a noninvasive imaging method allows differentiation between most benign and malignant focal liver lesions $[10,11]$. Only a few case reports have described the incidental findings [12] and CEUS features of focal PH. To the best of our knowledge, in this study, we report the largest series to date [13]. Our aim was to investigate the ultrasound and CEUS features of histopathologically proven focal $\mathrm{PH}$.

\section{Materials and Methods}

\section{Compliance with Ethical Standards}

The institutional review board of Zhongshan Hospital approved this retrospective study (ID: B2020-309R). Informed consent was waived before ultrasound examination. The procedure followed was in accordance with the Declaration of Helsinki.

\section{Patients}

Between January 2012 and May 2020, a multicenter retrospective study was performed among five institutions. Twenty-four patients with histopathologically confirmed PH (11 men, 13 women; mean age, $32.4 \pm 7.1$ years; range, 28 to 41 years) were analyzed (Table 1). All lesions were histologically proven, either by 18 - or 20-gauge core needle biopsy $(n=10)$ or by hepatic surgery $(n=14)$. All patients were examined with CEUS 2-3 days before ultrasound-guided core needle biopsy or liver resection. No exclusion criteria were defined.

\section{Ultrasound Examination Technique}

Conventional B-mode ultrasonography (BMUS) and CEUS were performed in all patients by sonologists with more than 10 years of liver CEUS experience. All ultrasound examinations were performed using one of four premium ultrasound systems: Acuson Sequoia (5C1 convex array probes, $3.5 \mathrm{MHz}, \mathrm{n}=3$, Siemens Healthineers, Mountain View, CA, USA), S2000 HELX OXANA (6C1 convex array probes, 3.5 MHz, $n=8$, Siemens Medical Solution USA Inc., Mountain View, CA, USA), Philips EPIQ7 (C2-9 convex array probes, 2-9 MHz, $n=6$, Philips, Bothell, WA, USA), LOGIQ E9 (C1-5 convex array probes, 1-5 MHz, $n=7$, GE Healthcare, Milwaukee, WI, USA).

SonoVue (sulfur hexafluoride, Bracco, Milan, Italy) was used with doses ranging between 1.5 and $2.4 \mathrm{~mL}$ depending on patient size and local practice. It was injected intravenously through a cannula placed in the antecubital fossa vein and immediately flushed with 5 $\mathrm{mL}$ of normal saline solution. Still images and videoclips, recorded for up to 3 minutes after contrast agent injection, were stored for

Table 1. Baseline characteristics of peliosis hepatis peliosis hepatis patients included in our study

\begin{tabular}{lc}
\multicolumn{1}{c}{ Characteristic } & No. $(\%)(n=24)$ \\
\hline Age (year) & $32.4 \pm 7.1$ \\
Mean士SD & $28-41$ \\
Range & $11 / 13$ \\
Male/Female & $17 / 7$ \\
No. of focal liver lesions (single/multiple) & \\
Presence of chronic liver disease & $4(16.7)$ \\
Hepatitis B & $1(4.2)$ \\
Alcoholic & \\
Histological results & $14(58.3)$ \\
Hepatic surgery & $10(41.7)$ \\
Core needle biopsy &
\end{tabular}

$\mathrm{PH}$, peliosis hepatis; SD, standard deviation. 
analysis.

\section{Imaging Analysis}

Two independent radiologists (with extensive CEUS experience of more than 10 years), who were blinded to the clinical and pathological data, reviewed all the stored anonymized ultrasound examinations. All CEUS features were interpreted according to the current WFUMB-EFSUMB guidelines $[10,11]$.

The reviewers documented the following BMUS features: maximum diameter of the focal liver lesion, echogenicity (homogeneous or heterogeneous; hyperechoic, hypoechoic, or isoechoic), shape (regular or irregular), margin (well or ill-defined), presence of color flow signal. The CEUS imaging review focused on the contrast enhancement pattern of lesion (hypo-enhanced, hyperenhanced, iso-enhanced, heterogeneous, and homogeneous) during the arterial (10-45 seconds), portal venous (30-120 seconds), and late (>120 seconds) phases of CEUS $[10,11]$.

\section{Histopathological Diagnosis}

Seven pathologists with hepatobiliary expertise reviewed all pathological slides. The final histopathological diagnosis was based on hematoxylin and eosin-stained sections and immunohistochemical staining results.

\section{Statistical Analysis}

Statistical analyses were performed using SPSS version 23.0 (IBM Corp., Armonk, NY, USA). The chi-square test and Fisher exact test were used to compare categorical parameters between two groups. Continuous parameters were presented as the mean \pm standard deviation, and the Student $t$ test was used. A P-value of less than 0.05 was considered to indicate statistical significance.

\section{Results}

\section{Epidemiological Data}

The epidemiological data of the study population are shown in Table 1.

\section{Clinical Findings}

The indications for imaging studies in patients were regular postoperative surveillance in five patients, preoperative staging of gastrointestinal cancer in three patients, screening for hepatocellular carcinoma (HCC) in four chronic hepatitis B patients, and regular health examinations for 12 patients. Serologic tumor markers including alpha-fetoprotein, carbohydrate antigen 19-9, and carcinoembryonic antigen were all within normal ranges. Peliosis was observed in three patients with concomitant hepatocellular adenoma, in two patients with HCC, and in 19 patients without a synchronous focal liver lesion.

\section{BMUS Features}

Single lesions were detected in 17 patients, while there were multiple lesions in seven patients. Most of the lesions (83.3\%, 20/24) were heterogeneously hypoechoic on BMUS, with welldefined margins but irregular shapes. No mass effect was evident in this group. Two lesions showed mixed echogenicity, but were predominantly hypoechoic. The mean size of all PH lesions was $36.8 \pm 12.4 \mathrm{~mm}$ (range, 10 to $80 \mathrm{~mm}$ ) and color Doppler flow signals were detected in $41.7 \%(10 / 24)$ of lesions. Calculations of the spectral Doppler trace showed a mean intra-lesional resistive index of $0.45 \pm 0.13$.

\section{CEUS Features}

CEUS was performed in all 24 patients diagnosed with PH. During the arterial phase, the lesions displayed either mild heterogeneous hyperenhancement $(83.3 \%, 20 / 24)$ or isoenhancement $(16.7 \%$, 4/24) compared to the surrounding liver parenchyma (Figs. 1, 2). In three cases, there was a progressive centrifugal enhancement pattern (Table 2). After 1 minute, in the portal venous phase, 21 lesions became mildly hypo-enhanced and washed out in the late phase. In our series there were three patients with phlebetactic $\mathrm{PH}$ who showed a strongly enhancing area in the central portion at the arterial phase, which then spread to the periphery in the progressively centrifugal direction during the portal venous and late phases (Fig. 3).

\section{Final Diagnosis}

Histopathological examinations with hematoxylin and eosin staining

Table 2. Contrast-enhanced ultrasound features of peliosis hepatis

\begin{tabular}{lc}
\hline \multicolumn{1}{c}{ Feature } & No. (\%) $(\mathrm{n}=24)$ \\
\hline Arterial phase & \\
Heterogeneous hyperenhancement & $20(83.3)$ \\
Centrifugal hyperenhancement & $3(12.5)$ \\
Isoenhancement & $4(16.7)$ \\
Portal venous phase & \\
Hyperenhancement & $3(12.5)$ \\
Isoenhancement & 0 \\
Hypoenhancement & $21(87.5)$ \\
Late phase & \\
Hyperenhancement & $3(12.5)$ \\
Isoenhancement & 0 \\
Hypoenhancement & $21(87.5)$ \\
\hline
\end{tabular}




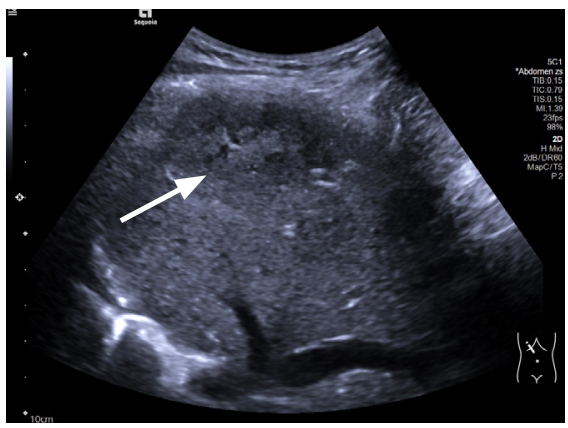

A

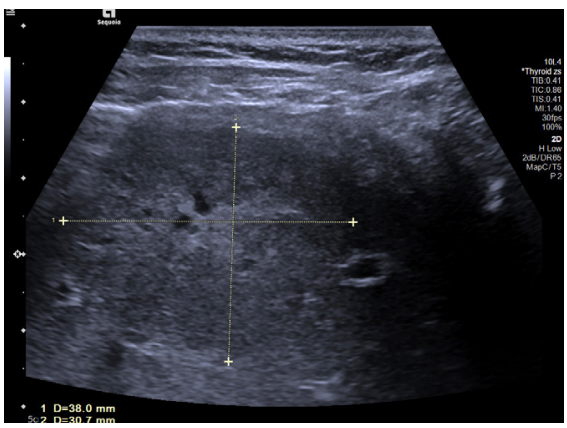

B

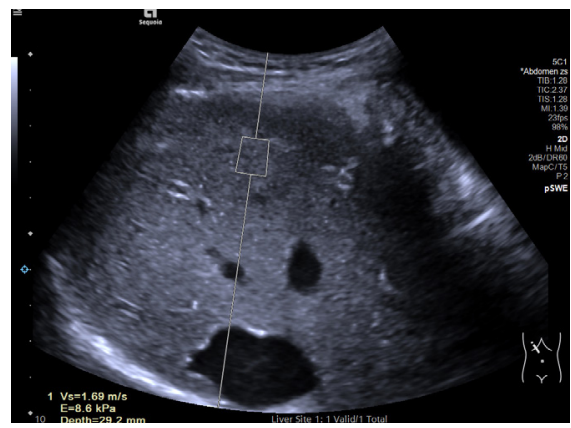

C

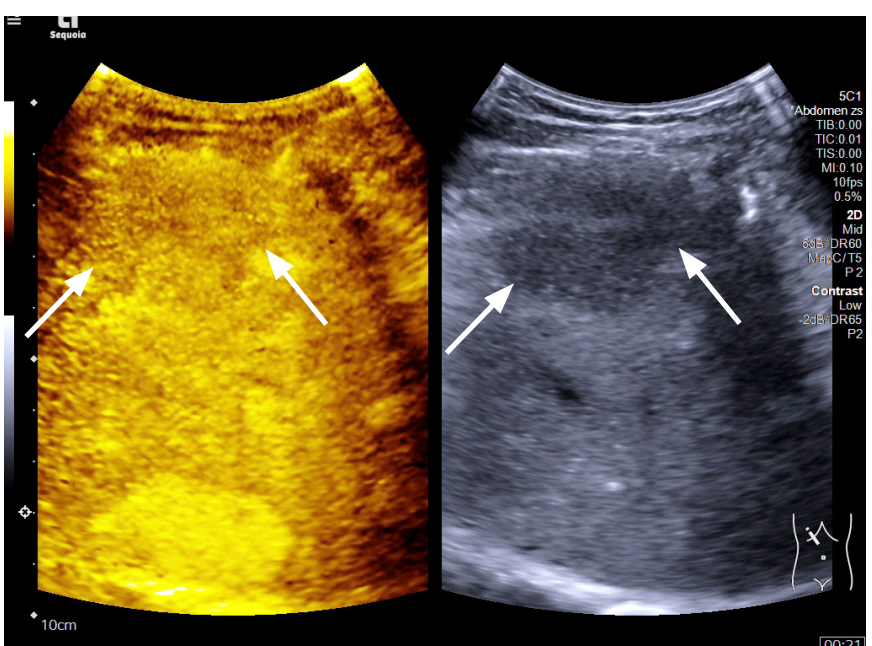

D

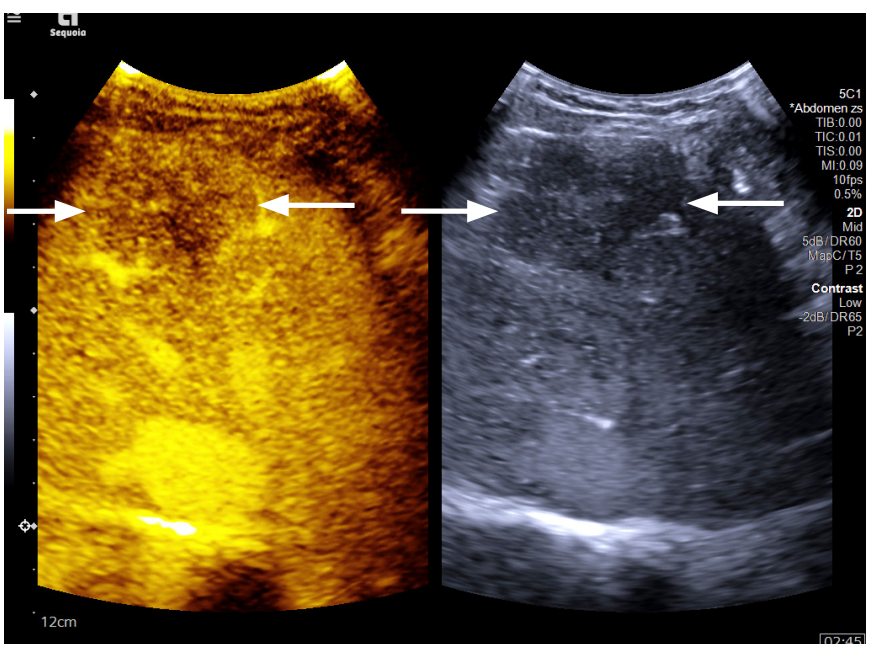

$\mathrm{F}$

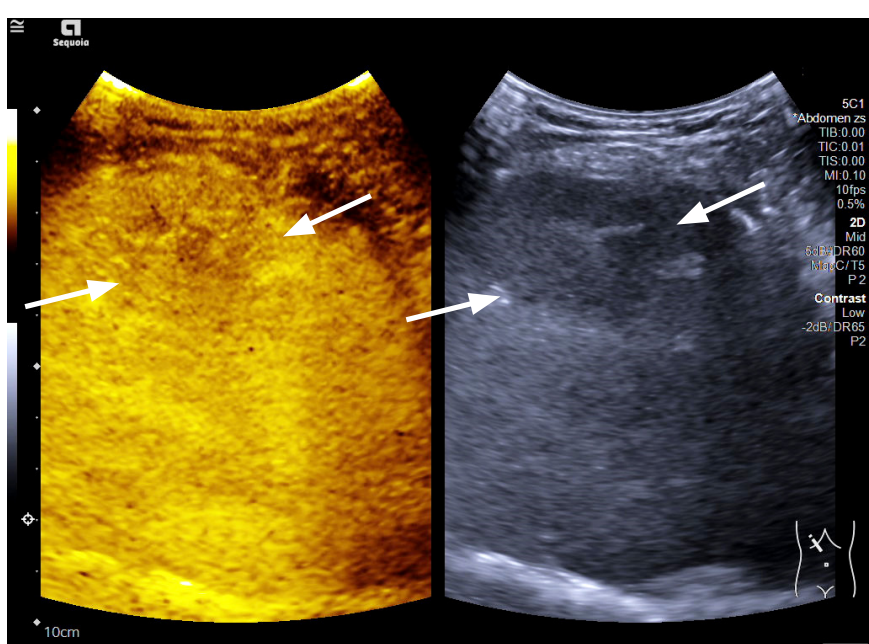

$\mathrm{E}$

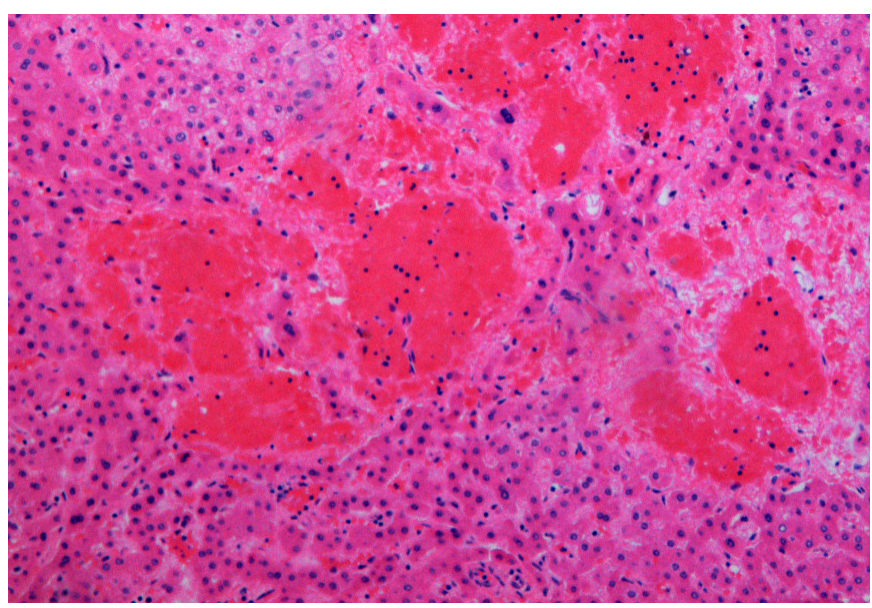

G

Fig. 1. Contrast enhanced ultrasound features of a focal peliosis hepatis.

A. B-mode ultrasonography (BMUS) shows a mixed-echogenicity and predominantly hypoechoic lesion (arrow) in the right lobe of the liver. B. The mass had a maximal diameter of $38 \mathrm{~mm}$ on BMUS measured using a linear transducer. C. Ultrasound shear wave elastography shows that the lesion was relatively soft compared to the surrounding liver parenchyma $(V s=1.69 \mathrm{~m} / \mathrm{s})$. D-F. On contrast-enhanced ultrasonography, the lesion shows heterogeneous isoenhancement (arrows) in the arterial phase (D), and hypoenhancement (arrows) in both the late portal venous phase (E) and late phase (F). G. Marked sinusoidal dilatation forming irregular spaces and thinning of hepatic cell cords are observed on the surgical resection specimen $(H \& E, \times 20)$. 


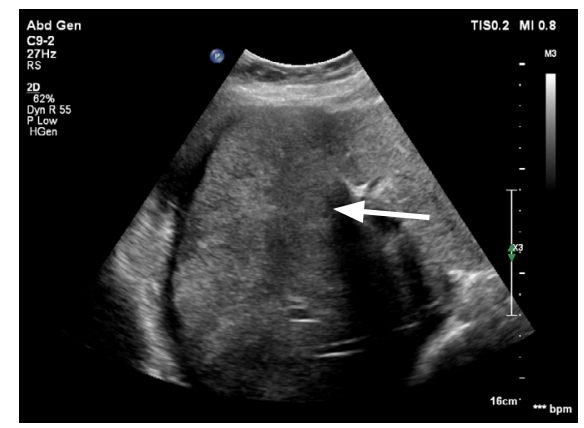

A

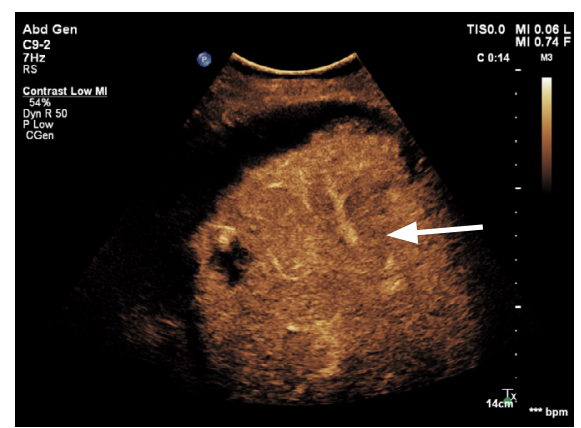

D

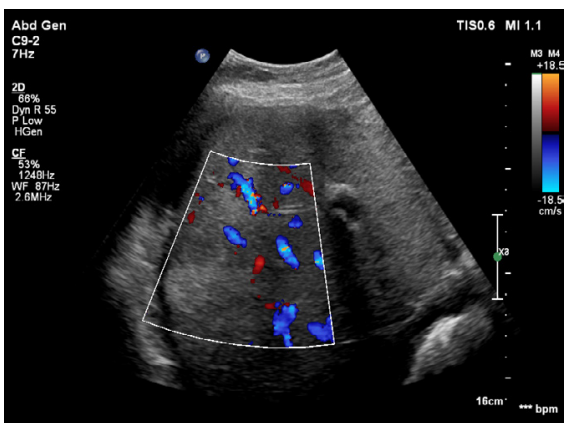

B

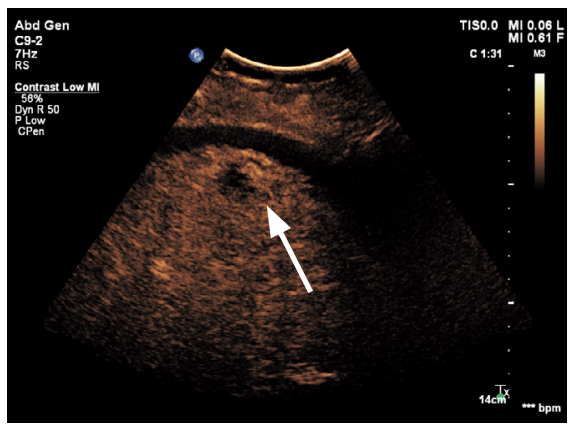

E

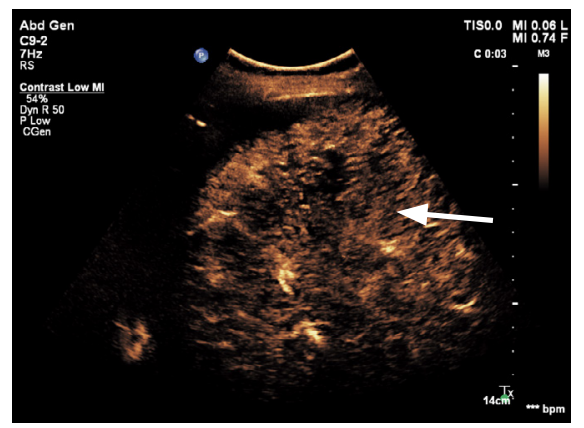

C

Fig. 2. Multilocular peliosis hepatis.

A. B-mode ultrasonography shows a slightly hypoechoic lesion (arrow) in the right lobe of the liver. B. Dotted color flow signals could be detected inside the lesion. C-E. After injection of contrast agents, multiple lesions could be detected as slightly hyperenhanced lesions during arterial phase (C), hypoe-enhanced during portal venou phase (D) and late phase (E) of contrast enhanced ultrasound. of the specimens revealed typical features of parenchymal PH in 21 patients, including the presence of localized irregular dilatation of sinusoids forming blood-filled spaces in the liver parenchyma, and thinning of hepatic cell cords and the reticulin fiber network. The presence of neoplastic cells was excluded (Figs. 1, 2).

In three patients with phlebetactic $\mathrm{PH}$, the presence of endothelial lining along the blood-filled spaces caused by aneurysmal dilatation of the central vein was observed.

\section{Discussion}

In this study, we analyzed the imaging features of histologicallyproven PH. Wagner first described PH in 1861. In 1916, Schoenlack termed it "peliosis," from the Greek word pelios, which means "livid" (i.e., discolored by extravasated blood) [14]. PH is a benign vascular condition with proliferating liver sinusoids resulting in cystic blood-filled cavities. These cysts communicate with the hepatic sinusoids and are associated with rupture of the reticulin framework [15]. The pathogenesis and etiology of $\mathrm{PH}$ remains unclear and is often unidentified in $20 \%-50 \%$ of patients [2]. It has been reported to be associated with chronic debilitating conditions (e.g., amyloidosis, chronic kidney diseases, infections, and immunocompromised status) and various drugs (e.g. steroids, oral contraceptives, and tamoxifen) [16]. In our results, there was no meaningful difference in the distribution of $\mathrm{PH}$ patients by sex.
$\mathrm{PH}$ may be circumscribed or form part of a focal liver lesion, but it can also present as a widespread, diffuse feature throughout the whole liver [17]. As we show in Table 1, most of the PH lesions in this series were single lesions, while seven presented as multiple lesions in the liver. The same process may occur in the spleen, bone marrow, lymph nodes, and more rarely in lungs, stomach, intestine, parathyroid, pancreas, pituitary gland, and kidneys $[14,16]$. The clinical presentation of $\mathrm{PH}$ may range from asymptomatic to progressive cholestasis, hepatic failure, and potentially spontaneous rupture that may be fatal $[2,6]$. In our current study, only five patients had a history of chronic liver disease.

The imaging findings of focal PH may vary depending on the size, pathological presentation, and stage of the lesion [6]. It has nonspecific features on conventional abdominal ultrasound and can present as a homogeneous, hypoechoic lesion in patients with fatty liver, a hyperechoic lesion in patients with a normal liver, or a heterogeneous lesion if complicated by hemorrhage [7]. Previous reports have described that the imaging features of focal $\mathrm{PH}$ can mimic a mass and may be difficult to distinguish from other hepatic lesions including hemangioma, HCC, metastases, adenoma, focal nodular hyperplasia (FNH), and multiple abscesses $[6,7,18,19]$. Most cases of $\mathrm{PH}$ are detected incidentally as a hypervascular tumor on cross-sectional imaging (CT/MRI, ultrasonography). $\mathrm{PH}$ may part of the histological features of hepatocellular adenoma [8,20], HCC [21], sarcoma, FNH [20], hemangioma [8], and other focal liver lesions 


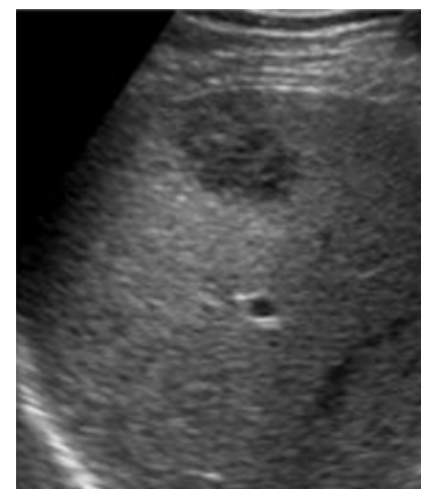

A

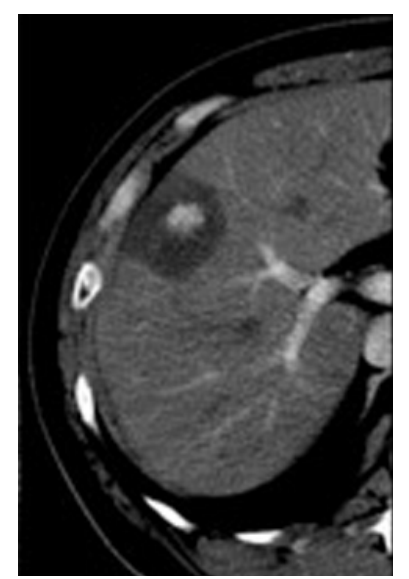

$E$

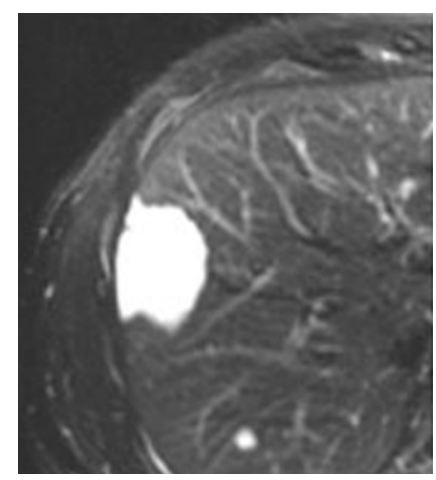

I

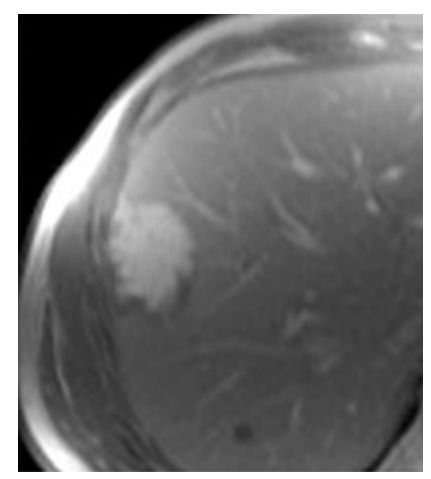

M

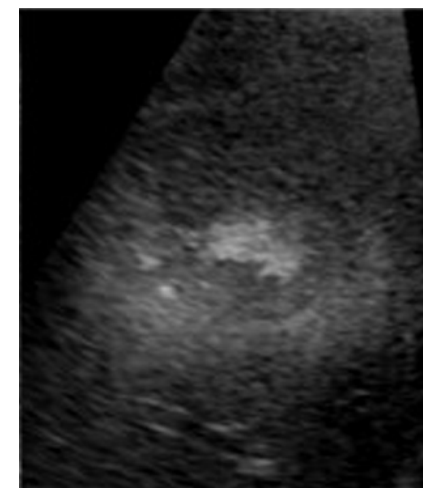

B

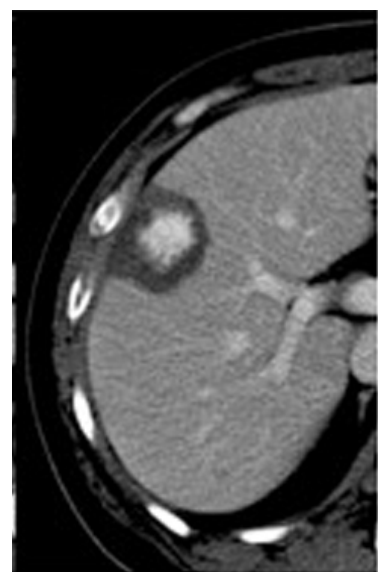

F

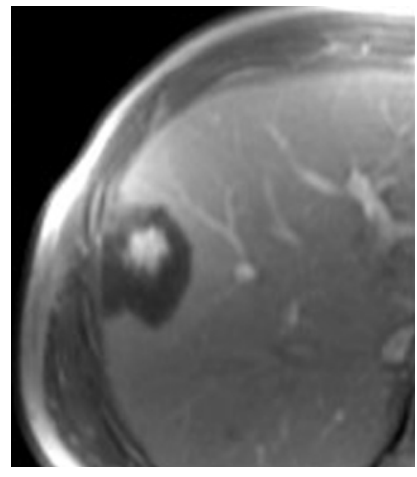

J

G

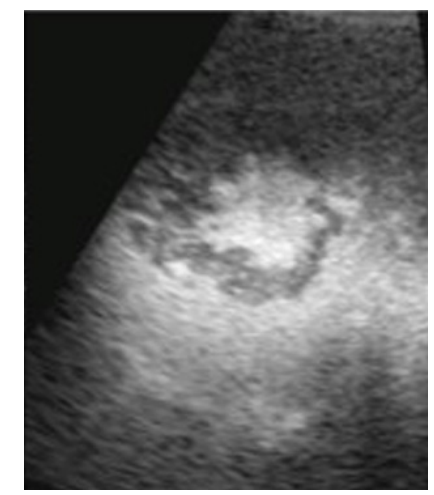

C
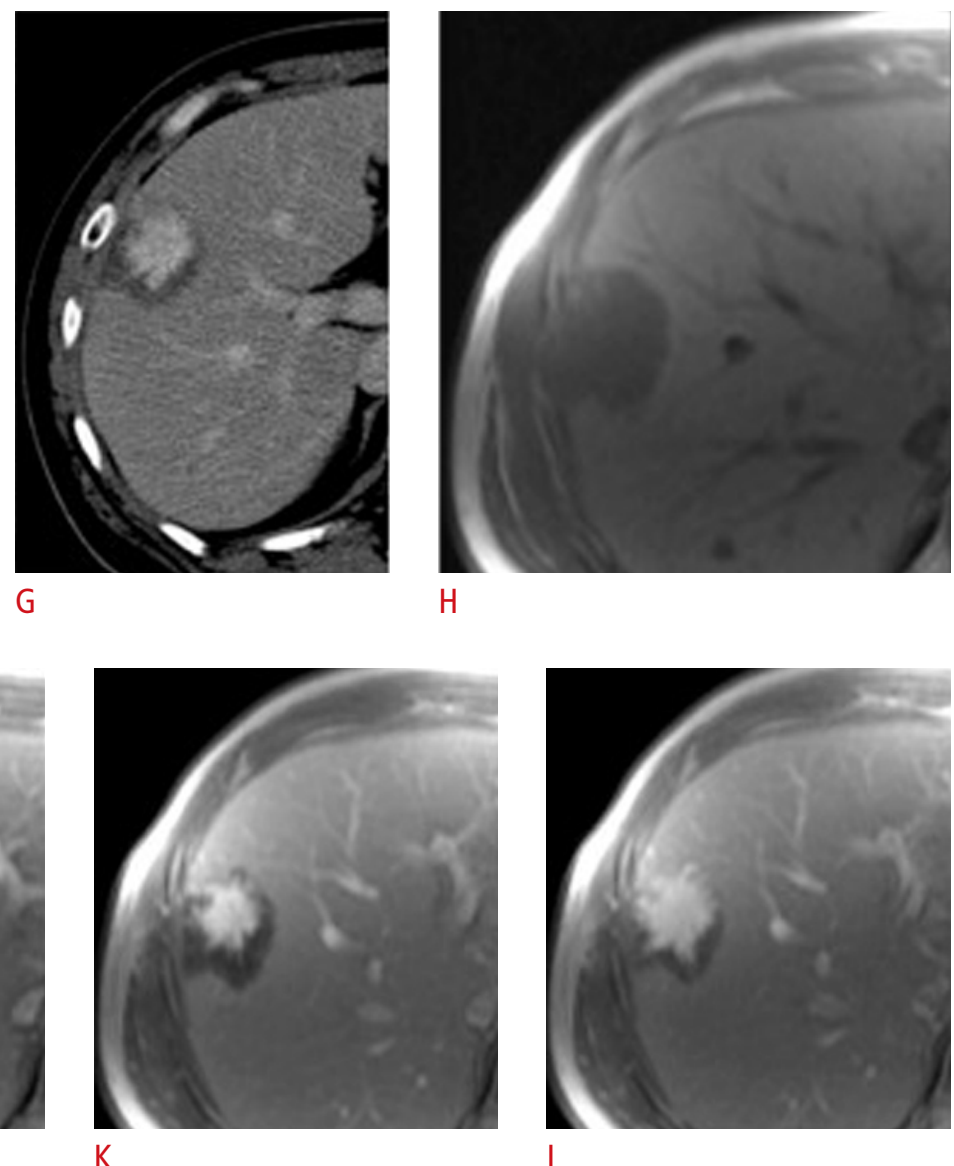

L

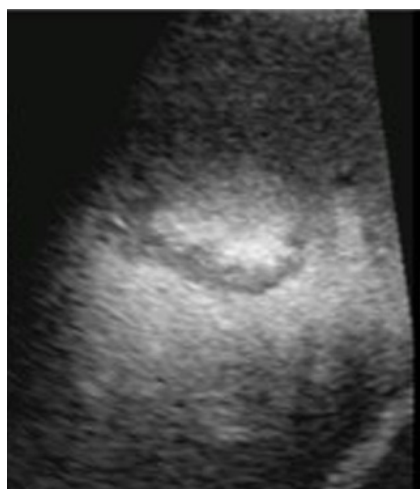

D

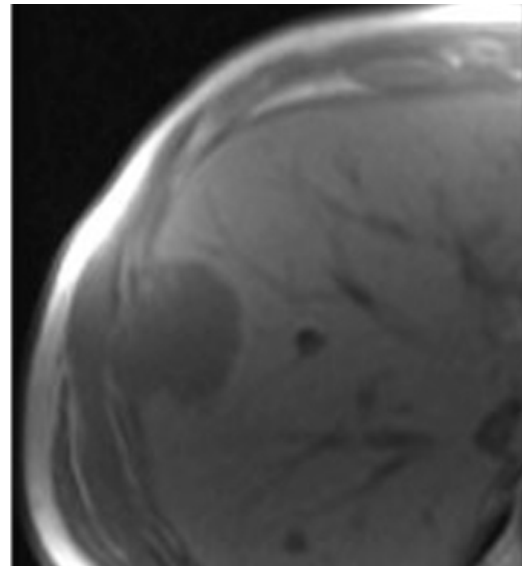

$\mathrm{H}$

Fig. 3. A 35-year-old man with peliosis hepatis of the phlebectatic type.

$A-D$. Contrast-enhanced ultrasound (CEUS) images obtained before contrast injection (A) and during the arterial (B), portal (C) and equilibrium (D) phases are shown. CEUS images show a strongly enhancing area in the central portion of the hypoechoic lesion in the arterial phase, which progressively spread to the periphery in a centrifugal direction during the portal and late equilibrium phases. $\mathrm{E}-\mathrm{G}$. Three-phase CT images obtained during the arterial (E), portal (F), and equilibrium (G) phases show the same hypoattenuating lesion with the same progressively centrifugal enhancement pattern in segment VIII. H, I. Pre-contrast T1-weighted (H) and T2-weighted (I) magnetic resonance (MR) images show the same lesion, which is hypointense on the T1-weighted images and hyperintense on the T2-weighted images. $J-M$. Post-contrast T1-weighted MR images obtained during the arterial $(J)$, portal $(K)$, equilibrium (L), and hepatobiliary (M) phases show the same hypointense lesion with the same progressively centrifugal enhancement pattern. 
including focal tuberculosis, as well as severe hepatic infections [19].

In this study, primary lesions did not exhibit a marked mass effect due to their diffuse characteristics, and most appeared as hypoechoic focal liver lesions (80.9\%). This is thought to correspond to the venous lakes that are commonly described histologically [7]. Two lesions were of mixed echogenicity, but predominantly hypoechoic; this may be due to the cystic cavities, thrombosis, or hemorrhage inside the vascular cavities of these lesions $[7,14]$. On color Doppler, it is possible to detect intra-lesional flow with typical low resistive indices on spectral Doppler [22]. Focal PH should be suspected when an incidentally detected focal liver lesion shows no specific ultrasound findings that clearly favor the diagnosis of a common tumor-like hepatic lesion [23]. The lack of a mass effect would be an even more strongly supportive finding.

The most common CEUS enhancement pattern described in our cases was mild heterogeneous hyperenhancement in the arterial phase, with washout as the most important CEUS feature in the late portal venous phase and late phase. These CEUS features of washout in the later phases are suggestive of the presence of non-hepatic tissue and thus warrant a further diagnostic work-up including a biopsy. It must be taken into account that hypoenhancement in the late phase is very rarely observed in CEUS examinations of benign focal liver lesions $[10,11]$.

The dynamic CEUS enhancement patterns of focal PH may also vary depending on the underlying disease and the various stages of the blood components [6]. Dynamic CEUS may thus show hypoenhancement of the lesion in the late portal venous phase and late phase [24]. A similar CEUS enhancement pattern was reported in a previous case report. In contrast, "atypical" enhancement patterns, such as a peripheral ring enhancement in the arterial phase with centripetal filling and homogeneous hyperenhancement in the late phase, have been described by Gronlykke et al. [25], while peripheral hyperenhancement with central but not peripheral washout in the late phase was described by Schuldes and Weickert [26]. Central enhancement probably reflects a similar pattern of blood flow reported on CT by Gouya et al. [27], which might be a potential differentiating feature from hepatic hemangiomas. Yanoff and Rawson [28] described two types of PH (parenchymal and phlebectatic), but imaging findings of the latter type have rarely been reported $[27,29]$. In addition, multilocular PH may be observed (Fig. 2).

In our series, there were three patients with phlebetactic $\mathrm{PH}$. The dynamic CEUS imaging features of these lesions showed a pathognomonic enhancement pattern with progressive centrifugal enhancement from the arterial into the late phase (Fig. 3). Pathologically, the main distinguishing feature of the phlebectatic $\mathrm{PH}$ subtype from the thrombotic PH subtype is the presence of endothelial lining along the blood-filled spaces, since it is known to be based on aneurysmal dilatation of the central vein $[27,29]$. This difference in blood supply is helpful to understand the hemodynamics and the "reversed" enhancement pattern of PH from hepatic hemangioma, which allows a clear differentiation between these two entities. The lack of any nodular peripheral hyperenhancement in the arterial phase of CEUS, as observed in our cases, would also be atypical for a hemangioma [5].

The imaging findings of $\mathrm{PH}$ in our study are somewhat similar to those described in hepatic tumors of various etiologies [6]. Thus, $\mathrm{PH}$ should always be considered in the differential diagnosis of focal liver lesions. The size of $\mathrm{PH}$ lesions ranges from a few millimeters to more than $4 \mathrm{~cm}$ [24], and PH appears to be a continuous process from diffuse infiltration to predominant focal infiltration. The hypoechoic images on BMUS, together with early arterial phase hyperenhancement on CEUS, may mimic HCC or liver metastasis [6]. However, the absence of a mass effect would be more suggestive of PH since peliosis presumably starts as a diffuse process. In addition, HCCs are usually hyper-enhanced in the arterial phase with relatively rapid washout in the portal venous phase compared to $\mathrm{PH}$. HCC is more common in the background of chronic hepatitis or liver cirrhosis. The enhancement features of HCC have been summarized by the Liver Imaging Reporting and Data System working group [30].

Although benign, asymptomatic, and usually an incidental finding, PH should always be kept in mind as a differential diagnosis of an atypical hypervascular hepatic lesion. The other differential diagnoses would include hepatic adenomas, FNH, Caroli disease, and multiple abscesses [7,9,24], although these may have individually distinguishing features. For example, when compared to $\mathrm{FNH}$, most PH lesions demonstrated heterogeneous hyperenhancement during the arterial phase of CEUS, rather than the typical avid homogeneous centrifugal pattern seen with FNH. All PH lesions also demonstrated hypoenhancement during the portal venous and late phases, while all FNH lesions typically show hyperenhancement or isoenhancement. By observing these typical CEUS features, it is possible to differentiate PH from FNH.

According to current guidelines, all focal liver lesions with washout on CEUS require a biopsy or excision for histological confirmation if not contraindicated $[10,11]$. Thus, a percutaneous biopsy is often necessary to reach a definitive diagnosis when $\mathrm{PH}$ is suspected on imaging studies [2] unless it is of the phlebetactic subtype, which has a pathognomonic CEUS enhancement pattern.

There are some limitations of our study. Owing to the rarity of this disease, the predominant limitation is the small sample size. However, to the best of our knowledge, this is the largest series of $\mathrm{PH}$ described in the literature. Further large-scale research will be needed to validate the ultrasound features described herein. 
In addition, as this was a retrospective study, various ultrasound scanners and transducers were utilized and only patients with histologically-confirmed PH were included.

In conclusion, the CEUS characteristics of focal PH are typically the lack of a mass effect on BMUS, and mild heterogeneous arterial hyperenhancement and washout in the portal venous and late phases. In the absence of malignancy, PH should be considered, particularly if the imaging characteristics are not specific for a known common hepatic lesion.

ORCID: Yi Dong: https://orcid.org/0000-0002-0212-1477; Wen-Ping Wang: https:// orcid.org/0000-0002-6797-1835; Adrian Lim: https://orcid.org/0000-0003-39042876; Won Jae Lee: https://orcid.org/0000-0001-5833-989X; Dirk-Andre Clevert: https://orcid.org/0000-0003-3889-5447; Michael Höpfner: https://orcid.org/00000003-4538-2869; Andrea Tannapfel: https://orcid.org/0000-0002-0711-7100; Christoph Frank Dietrich: https://orcid.org/0000-0001-6015-6347

\section{Author Contributions}

Conceptualization: Dong Y, Wang WP, Lim A, Lee WJ, Clevert DA, Höpfner M, Tannapfel A, Dietrich CF. Data acquisition: Dong Y, Wang WP, Lim A, Lee WJ, Clevert DA, Höpfner M, Tannapfel A, Dietrich CF. Data analysis or interpretation: Dong Y, Wang WP, Lim A, Lee WJ, Clevert DA, Höpfner M, Tannapfel A, Dietrich CF. Drafting of the manuscript: Dong Y, Wang WP, Lim A, Lee WJ, Clevert DA, Höpfner M, Tannapfel A, Dietrich CF. Critical revision of the manuscript: Dong Y, Wang WP, Lim A, Lee WJ, Clevert DA, Höpfner M, Tannapfel A, Dietrich CF. Approval of the final version of the manuscript: all authors.

\section{Conflict of Interest}

No potential conflict of interest relevant to this article was reported.

\section{References}

1. Iwata T, Adachi K, Takahashi M. Peliosis hepatis mimicking malignant hypervascular tumors. J Gastrointest Surg 2017;21:10951098.

2. Iannaccone R, Federle MP, Brancatelli G, Matsui O, Fishman EK, Narra VR, et al. Peliosis hepatis: spectrum of imaging findings. AJR Am J Roentgenol 2006;187:W43-W52.

3. Levin D, Hod N, Anconina R, Ezroh Kazap D, Shaco-Levy R, Lantsberg $S$. Peliosis hepatis simulating metastatic liver disease on FDG PET/CT. Clin Nucl Med 2018;43:e234-e236.

4. Braden B, Helm B, Fabian T, Dietrich CF. Bacillary angiomatosis of the liver, a suspected ultrasound diagnosis? Z Gastroenterol 2000;38:785-789.

5. Crocetti D, Palmieri A, Pedulla G, Pasta V, D'Orazi V, Grazi GL.
Peliosis hepatis: personal experience and literature review. World J Gastroenterol 2015;21:13188-13194.

6. Kim SH, Lee JM, Kim WH, Han JK, Lee JY, Choi BI. Focal peliosis hepatis as a mimicker of hepatic tumors: radiological-pathological correlation. J Comput Assist Tomogr 2007;31:79-85.

7. Ferrozzi F, Tognini G, Zuccoli G, Cademartiri F, Pavone P. Peliosis hepatis with pseudotumoral and hemorrhagic evolution: CT and MR findings. Abdom Imaging 2001;26:197-199.

8. Dietrich CF, Tana C, Caraiani C, Dong Y. Contrast enhanced ultrasound (CEUS) imaging of solid benign focal liver lesions. Expert Rev Gastroenterol Hepatol 2018;12:479-489.

9. Slim R, Hachem N, Smayra V, Yaghi C, Daniel F, Boujaoude J, et al. Education and Imaging. Hepatobiliary and pancreatic: peliosis hepatis associated with multiple myeloma. J Gastroenterol Hepatol 2014;29:5.

10. Dietrich CF, Nolsoe CP, Barr RG, Berzigotti A, Burns PN, Cantisani $V$, et al. Guidelines and good clinical practice recommendations for contrast enhanced ultrasound (CEUS) in the liver: update 2020: WFUMB in cooperation with EFSUMB, AFSUMB, AIUM, and FLAUS. Ultraschall Med 2020;41:562-585.

11. Dietrich CF, Nolsoe CP, Barr RG, Berzigotti A, Burns PN, Cantisani $V$, et al. Guidelines and good clinical practice recommendations for contrast-enhanced ultrasound (CEUS) in the liver: update 2020 WFUMB in cooperation with EFSUMB, AFSUMB, AIUM, and FLAUS. Ultrasound Med Biol 2020;46:2579-2604.

12. Dietrich CF, Westerway S, Nolsoe C, Kim S, Jenssen C. Commentary on the World Federation for Ultrasound in Medicine and Biology project "Incidental Findings". Ultrasound Med Biol 2020;46:18151820.

13. Loizides A, Glodny B, Zoller H, Zelger BG, Junker D, Henninger B, et al. Contrast enhanced ultrasound of a rare case of peliosis hepatis. Med Ultrason 2017;19:114-116.

14. Yu CY, Chang LC, Chen LW, Lee TS, Chien RN, Hsieh MF, et al. Peliosis hepatis complicated by portal hypertension following renal transplantation. World J Gastroenterol 2014;20:2420-2425.

15. Oriordan K, Blei A, Vogelzang R, Nemcek A, Abecassis M. Peliosis hepatis with intrahepatic hemorrhage: successful embolization of the hepatic artery. HPB Surg 2000;11:353-358.

16. Choi SK, Jin JS, Cho SG, Choi SJ, Kim CS, Choe YM, et al. Spontaneous liver rupture in a patient with peliosis hepatis: a case report. World J Gastroenterol 2009;15:5493-5497.

17. Ben Hassen $W$, Wagner $M$, Lucidarme O. Unusual hepatic lesion in a patient with a lung tumor. Gastroenterology 2014;147:e7-e8.

18. Dong Y, Teufel A, Wang WP, Dietrich CF. Current opinion about hepatocellular carcinoma $<10 \mathrm{~mm}$. Digestion 2021;102:335-341.

19. Dietrich CF, Douira-Khomsi W, Gharbi H, Sharma M, Cui XW, Sparchez Z, et al. Cystic and alveolar echinococcosis of the hepatobiliary tract: the role of new imaging techniques for improved diagnosis. Med Ultrason 2020;22:75-84. 
20. Khanna M, Ramanathan S, Fasih N, Schieda N, Virmani V, Mclnnes MD. Current updates on the molecular genetics and magnetic resonance imaging of focal nodular hyperplasia and hepatocellular adenoma. Insights Imaging 2015;6:347-362.

21. Hoshimoto S, Morise Z, Suzuki K, Tanahashi Y, Ikeda M, Kagawa T, et al. Hepatocellular carcinoma with extensive peliotic change. J Hepatobiliary Pancreat Surg 2009;16:566-570.

22. Hocke M, Ignee A, Topalidis T, Stallmach A, Dietrich CF. Contrastenhanced endosonographic Doppler spectrum analysis is helpful in discrimination between focal chronic pancreatitis and pancreatic cancer. Pancreas 2007;35:286-288.

23. Ignee A, Gebel M, Caspary WF, Dietrich CF. Doppler imaging of hepatic vessels: review. Z Gastroenterol 2002;40:21-32.

24. Fowell AJ, Mazhar D, Shaw AS, Griffiths WJ. Education and imaging: hepatobiliary and pancreatic: peliosis hepatis. J Gastroenterol Hepatol 2011;26:1082.

25. Gronlykke L, Tarp B, Dutoit SH, Wilkens R. Peliosis hepatis: a complicating finding in a case of biliary colic. BMJ Case Rep 2013;2013:bcr2013200539.

26. Schuldes M, Weickert U. Contrast-enhanced ultrasound in suspected liver metastases. Dtsch Med Wochenschr 2011;136:1255-1256.

27. Gouya H, Vignaux O, Legmann P, de Pigneux G, Bonnin A. Peliosis hepatis: triphasic helical CT and dynamic MRI findings. Abdom Imaging 2001;26:507-509.

28. Yanoff M, Rawson AJ. Peliosis hepatis. an anatomic study with demonstration of two varieties. Arch Pathol 1964;77:159-165.

29. Tsukamoto $Y$, Nakata H, Kimoto T, Noda T, Kuroda Y, Haratake J. CT and angiography of peliosis hepatis. AJR Am J Roentgenol 1984; 142:539-540.

30. Dietrich CF, Potthoff A, Helmberger T, Ignee A, Willmann JK; CEUS LI-RADS Working Group. Contrast-enhanced ultrasound: Liver Imaging Reporting and Data System (CEUS LI-RADS). Z Gastroenterol 2018;56:499-506. 